Proceeding Series of the Brazilian Society of Computational and Applied Mathematics

\title{
Estudo da equação de Fokker-Planck para os potenciais de Hulthén e de Morse generalizado via supersimetria
}

\author{
Rita de Cássia dos Anjos ${ }^{1}$ \\ Universidade Federal do Paraná, UFPR, Palotina, PR \\ Gisele Bosso de Freitas ${ }^{2}$ \\ Departamento de Bioestatística, IBB-UNESP, Botucatu, SP
}

Resumo. Neste trabalho utiliza-se o formalismo da supersimetria em mecânica quântica para obter e estudar o comportamento das soluções analíticas da equação de Fokker-Planck para os potenciais de Hulthén e de Morse generalizado.

Palavras-chave. Fokker-Planck, Schrödinger, Hulthén, Morse generalizado, potencial.

\section{Métodos e Resultados}

Os potenciais de Hulthén [2] e de Morse generalizado [1] são bastante utilizados no contexto biofísico, o potencial de Hulthén, por exemplo, é utilizado para descrever interações atômicas com aplicações em física nuclear, em física do estado sólido e em fisico-química e o potencial de Morse generalizado, descreve moléculas diatômicas e modelos físicos para o DNA [3].

O formalismo da supersimetria em mecânica quântica [7], basicamente consiste em, dado um hamiltoniano da equação de Schrödinger, substituí-lo pela combinação de dois operadores diferenciais de primeira ordem. Então, dado um operador diferencial de segunda ordem (hamiltoniano), temos que encontrar dois operadores $[5,6]$ de forma a transformar uma equação diferencial parcial em uma equação diferencial ordinária e dessa forma encontrar as autofunções e autovalores que caracterizam o sistema estudado. Matematicamente, dado

$$
H_{1}=\frac{\hbar^{2}}{2 m} \frac{d^{2}}{d x^{2}}+V_{1}(x)=a_{1}^{+} a_{1}^{-}+E_{0}^{(1)},
$$

em que $a^{ \pm}$chamados de operadores escada,são dados por

$$
a^{ \pm}=\mp \frac{d}{d x}+W_{1}(x)
$$

\footnotetext{
${ }^{1}$ ritacassia@ufpr.br

${ }^{2}$ freitas.gibb@gmail.com
} 
e $W_{1}(x)$ é o superpotencial. A solução geral é dada por,

$$
\begin{aligned}
H_{n} & =a_{n}^{+} a_{n}^{-}+E_{0}^{n}, \\
a_{n}^{ \pm} & =\mp \frac{d}{d x}+W_{n}(x), \\
\Psi_{n}^{(1)} & =a_{1}^{+} a_{2}^{+} \ldots a_{n}^{+} \psi_{0}^{(n+1)}, E_{n}^{(1)}=E_{0}^{(n+1)} .
\end{aligned}
$$

A equação de Fokker-Planck descreve a evolução temporal da distribuição de probabilidade $P(x, t)$ :

$$
\frac{\partial}{\partial t} P(x, t)=\frac{\partial}{\partial x}[f(x, t) P(x, t)]+\frac{\Gamma}{2} \frac{\partial^{2}}{\partial x^{2}} P(x, t),
$$

em que $\Gamma$ é a constante de difusão e $f(x)$ é a função associada ao potencial $V(x)$. Uma das maneiras de se obter a solução para a equação de Fokker-Planck é associando-a com a equação de Schrödinger, o que é bem estabelecido na literatura $[4,8]$. A associação entre as equações de Fokker-Planck e Schrödinger indica que as soluções de cada uma delas pode ser obtida através da outra.

As soluções analíticas obtidas utilizando o método mostram as distribuições de probabilidade para cada potencial, indicando que o comportamento de um sistema estocástico descrito através da equação de Fokker-Planck com o potencial de Morse generalizado tem a forma de uma curva normal e para o potencial de Hulthén, de uma curva assimétrica positiva. É importante ressaltar que apesar da dependência temporal, a forma geral não é afetada.

\section{Referências}

[1] A. Del Sol Mesa et al, Generalized Morse potential: Symmetry and satellite potentials, J. Phys. A: Math. Gen., vol. 31, 321-335, (1998).

[2] E. Drigo Filho, R. M. Ricotta, Supersymmetry, variational method and Hulthén potential, Mod. Phys. Let. A, World Scientific Publishing Co, vol. 10, 1613, (1995).

[3] M. Peyrard, A. R. Bishop, Statistical mechanics of a nonlinear model for DNA denaturation,Phys. Rev. Lett., vol. 62, 2755, (1989).

[4] H. Risken, The Fokker-Planck Equation, New York, Springer, (1984).

[5] J. O. Rosas-Ortiz, On the factorization method in quantum mechanics, (A. Ballestrero, et al, eds.), 285-299, Burgos, Spain, (1999).

[6] E. Schrödinger, A method of determining quantum-mechanical eigenvalues and eigenfunctions, Proc. Roy. Irish Acad. A, vol. 46, 9-16, (1940).

[7] G. Stedman, Simple supersymmetry: II. Factorisation method in quantum mechanics, Eur. J. Phys. vol. 6, 225-231 (1985).

[8] T. Tomé, T., M. J. Oliveira, Dinâmica Estocástica e Irreversibilidade, São Paulo, Edusp, (2001). 